\title{
N1-guanyl-1, 7-diaminoheptane enhances the sensitivity of pancreatic ductal adenocarcinoma cells to gemcitabine via the inhibition of eukaryotic translation initiation factor $5 \mathrm{~A} 2$
}

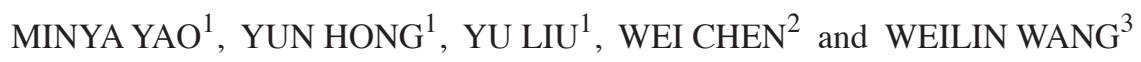 \\ ${ }^{1}$ Department of Breast Surgery, The First Affiliated Hospital, School of Medicine, Zhejiang University, \\ Hangzhou, Zhejiang 310003; ${ }^{2}$ Department of Surgery, The Second Affiliated Hospital, School of Medicine, \\ Zhejiang University, Hangzhou, Zhejiang $310009 ;{ }^{3}$ Department of Hepatobiliary and Pancreatic Surgery, \\ Collaborative Innovation Center for Diagnosis and Treatment of Infectious Diseases, The First Affiliated \\ Hospital, School of Medicine, Zhejiang University, Hangzhou, Zhejiang 310003, P.R. China
}

Received November 1, 2016; Accepted April 7, 2017

DOI: $10.3892 / \mathrm{etm} .2017 .4740$

\begin{abstract}
Pancreatic ductal adenocarcinoma (PDAC) is a highly aggressive malignancy due to its broad resistance to chemotherapy. Gemcitabine is used as a standard chemotherapeutic drug for PDAC treatment, either alone or in combination with other chemotherapeutics. However, in patients with advanced disease, survival is rarely improved. This study aimed to investigate the therapeutic efficacy of N1-guanyl-1, 7-diaminoheptane (GC7) combined with gemcitabine in PDAC therapy. We measured eukaryotic translation initiation factor 5A2 (eIF5A2) expression and gemcitabine sensitivity in different PDAC cell lines (Panc-1, BxPC-3, and T3-M4). The synergistic cytotoxic effects of gemcitabine combined with GC7 were measured using Cell Counting Kit-8 assays. Western blots were performed to measure eIF5A2 and multi-drug resistance 1 (MDR1) protein expression in PDAC cells. The present findings demonstrated that combined treatment with GC7 and gemcitabine significantly inhibited PDAC cell line viability $(\mathrm{P}<0.05)$. EdU incorporation assays also indicated that GC7 co-treatment remarkably enhanced gemcitabine sensitivity in PDAC cells. Furthermore, downregulation of eIF5A2 diminished the regulatory role of GC7 in gemcitabine cytotoxicity. Western blotting data indicated that GC7 downregulated the expression of MDR1 while gemcitabine induced MDR1 upregulation. These findings showed that GC7 combination
\end{abstract}

Correspondence to: Dr Weilin Wang, Department of Hepatobiliary and Pancreatic Surgery, Collaborative Innovation Center for Diagnosis and Treatment of Infectious Diseases, The First Affiliated Hospital, School of Medicine, Zhejiang University, 79 Qingchun Road, Hangzhou, Zhejiang 310003, P.R. China

E-mail: wangweilin6781@163.com

Key words: pancreatic ductal adenocarcinoma, gemcitabine, eukaryotic translation initiation factor 5A2, drug resistance, N1-guanyl-1, 7-diaminoheptane therapy may enhance the therapeutic efficacy of gemcitabine in PDAC by downregulating MDR1 expression.

\section{Introduction}

Pancreatic ductal adenocarcinoma (PDAC) is considered the fourth leading cause of cancer-associated mortality and is considered to be a malignancy with particularly high morbidity and mortality rates (1). Indeed, the 5-year survival rate of $<5 \%$ is due to the high probability of PDAC recurrence, despite the availability of multimodal treatments (2-4). It is projected that pancreatic cancer-related mortality rates will increase and surpass those of breast, prostate, and colorectal cancer by 2030 (5). As a standard chemotherapy, gemcitabine alone or in combination with other chemotherapeutics has demonstrated improvement in overall survival (6). However, disease control rates and survival remain far from satisfactory. Therefore, it is imperative that drug resistance mechanisms to gemcitabine are identified, which may help to generate more effective therapies for PDAC.

Eukaryotic translation initiation factor 5A2 (eIF5A2) from chromosome $3 \mathrm{q} 26$ in ovarian carcinoma, is one of the two isoforms from the eIF5A2 family, and has been reported to possess anti-tumor potential in various types of human cancer (7). Numerous studies have indicated that eIF5A2 is overexpressed in human cancer, including pancreatic ductal adenocarcinoma $(8,9)$, ovarian cancer (10), hepatocellular carcinoma (11,12), non-small cell lung cancer (13), esophageal squamous cell carcinoma (14), gastric cancer (15), colorectal cancer (16), and melanoma (17). Furthermore, eIF5A2 has been demonstrated to be useful as a biomarker for predicting the prognosis of certain types of cancer (18). Furthermore, inhibition of eIF5A2 by N1-guanyl-1, 7-diaminoheptane (GC7), an inhibitor of deoxyhypusine synthase, has been shown to enhance anti-proliferative and anti-tumor effects in human cancer cells $(7,19)$. However, the relationship between eIF5A2 and GC7 on PDAC drug resistance has never been investigated. Therefore, the present study aimed to investigate 
the therapeutic effect of GC7 combined with gemcitabine in PDAC cell therapy.

The present study aimed to investigate the relationship between the expression of eIF5A2 and drug resistance, the cell viability of gemcitabine treatment alone or combined with GC7 and the correlation of GC7 and multi-drug resistance 1 (MDR1) expression in human PDAC cell lines.

\section{Materials and methods}

Cell culture and reagents. Human PDAC cell lines (Panc-1, BxPC-3 and T3-M4) were obtained from American Type Culture Collection (Manassas, VA, USA). Panc-1, BxPC3 and T3-M4 cells were maintained in RPMI-1640 containing 10\% fetal bovine serum (FBS; Gibco; Thermo Fisher Scientific, Inc., Waltham, MA, USA) and $1 \%$ penicillin/streptomycin (Sigma-Aldrich; Merck KGaA, Darmstadt Germany). All cells were cultured in $5 \% \mathrm{CO}_{2}$ at $37^{\circ} \mathrm{C}$ in a humidified incubator. GC7 was purchased from Merck \& Co., Inc., (Darmstadt, Germany). Gemcitabine was obtained from Eli Lilly and Co., (Indianapolis, IN, USA). eIF5A2 siRNA and negative siRNA materials were purchased from Santa Cruz Biotechnology, Inc., (Santa Cruz, CA, USA). Anti-eIF5A2 antibody was obtained from Abcam (Cambridge, MA, USA; ab150439).

siRNA transfection. Pancreatic cancer cells were seeded onto 6-well plates at a density of $2 \times 10^{5}$ cells/well. When cells reached $60-70 \%$ confluence, cells were transfected with eIF5A2 siRNA or negative siRNA using Lipofectamine 2000 according to the manufacturer's protocol (Thermo Fisher Scientific, Inc.). Opti-MEM transfection medium (Gibco; Thermo Fisher Scientific, Inc.) was replaced with complete medium $6 \mathrm{~h}$ after transfection and the cells were incubated for $24 \mathrm{~h}$. The effect of eIF5A2 transfection was subsequently measured by western blotting.

Cell viability assays. Pancreatic cancer cells were seeded onto 96-well plates at a density of $4 \times 10^{3}$ cells/well. All cells were maintained in RPMI-1640 supplemented with 10\% FBS and $1 \%$ penicillin/streptomycin. After cells had attached to the flask, the medium was replaced with the corresponding $1 \%$ FBS medium for $24 \mathrm{~h}$ to allow synchronization of the cell cycle. The medium was then replaced with complete medium containing various drugs concentrations $(0,2,4,8,16,32$ and $64 \mu \mathrm{M}$ of GC7 was used; for Panc-1 and T3-M4, concentrations of $0,2,4,8,16,32$ and $64 \mu \mathrm{g} / \mathrm{ml}$ gemcitabine were used whereas for BxPC-3, concentrations of $0,0.5,1,2,4,8$ and $16 \mu \mathrm{g} / \mathrm{ml}$ were used) for $48 \mathrm{~h}$. Following this, $10 \mu \mathrm{l} /$ well CCK-8 solution (Dojindo Molecular Technologies, Inc., Kumamoto, Japan) was added and incubated with the plates for $3 \mathrm{~h}$ at $37^{\circ} \mathrm{C}$, and the optical density (OD) was determined at $450 \mathrm{~nm}$ with an MRX II microplate reader (Dynex Technologies, Inc., Chantilly, VA, USA). Furthermore, the half maximal inhibitory concentration $\left(\mathrm{IC}_{50}\right)$ was calculated.

Western blot analysis. Cells were harvested and lysed in cell lysis buffer containing protease inhibitors in an ice bath. Protein concentration was determined by a BCA Protein Assay Kit (Sigma-Aldrich; Merck KGaA). Samples of $\sim 20 \mu \mathrm{g}$ protein were separated by $10 \%$ SDS-PAGE and transferred to polyvinylidene difloride membranes (EMD Millipore, Billerica, MA, USA). Membranes were then blocked with Tris-buffered saline and $0.1 \%$ Tween 20 (TBST) containing $5 \%$ bovine serum albumin (Sigma-Aldrich; Merck KGaA, Darmstadt Germany) before incubation with anti-eIF5A2 primary antibody (diluted $1: 1,000$ in TBST) overnight at $4^{\circ} \mathrm{C}$. Following washing three times with TBST, the membranes were incubated with a horseradish peroxidase-labeled secondary antibody (diluted 1:2,000; Abcam, Cambridge, MA, USA; ab97051) for $2 \mathrm{~h}$ at $37^{\circ} \mathrm{C}$. Membranes were detected by chemiluminescence and protein bands were visualized by autoradiography (Kodak Corp., Rochester, NY, USA). GAPDH (diluted 1:5,000 in TBST; Cell Signaling Technology, Inc., Danvers, MA, USA; 5174S) was used as an internal control.

RNA extraction and reverse transcription-quantitative polymerase chain reaction (RT-qPCR) analysis. Human pancreatic cancer cell lines were extracted using TRIzol reagent (Thermo Fisher Scientific, Inc.) according to the manufacturer's instructions. Reverse transcription into cDNA using Prime Script reagent RT Kit (Takara Biotechnology Co., Ltd., Dalian, China) was performed and the following thermocycling conditions were used: $15 \mathrm{~min}$ at $37^{\circ} \mathrm{C}$ and $5 \mathrm{sec}$ at $85^{\circ} \mathrm{C}$. According to the manufacturer's instructions, SYBR Premix Ex Taq (Tli RNaseH Plus; Takara Biotechnology Co., Ltd., Dalian, China; RR420A) qPCR was performed at $50^{\circ} \mathrm{C}$ for $2 \mathrm{~min}$ and $95^{\circ} \mathrm{C}$ for $30 \mathrm{sec}$, followed by 40 cycles of denaturation at $95^{\circ} \mathrm{C}$ for $15 \mathrm{sec}$, and annealing at $60^{\circ} \mathrm{C}$ for $30 \mathrm{sec}$. All reactions were performed in triplicate. Expression of eIF5A2 mRNA was normalized to GAPDH (housekeeping control) (both Takara Biotechnology Co., Ltd.). Primer sequences were as follows: eIF5A2 forward 5'-TATGCAGTGCTCGGCCTT G-3' and reverse 5'-TTGGAACATCCATGTTGTGAGTAG A-3'; and GAPDH forward 5'-CGGAGTCAACGGATTTGG TCGTAT-3' and reverse 5'-AGCCTTCTCCATGGTGGTGAA GAC-3'. The average $\mathrm{Cq}$ value (amplified power curve inflection point) was read. Calculation of the relative expression of the target gene wasperformed using the $2^{-\Delta \Delta \mathrm{Cq}}$ method (20).

Statistical analysis. Experimental results were expressed as the mean \pm standard deviation. Statistical analysis was performed using Prism 5 (GraphPad Software, Inc., San Diego, CA, USA). Statistical differences between two groups were examined with the Student's t-test and multiple groups were compared using one-way analysis of variance. $\mathrm{P}<0.05$ was considered to indicate a statistically significant difference.

\section{Results}

Gemcitabine sensitivities in PDAC cell lines. To evaluate the effect of gemcitabine in pancreatic cancer cells, a CCK-8 assay was used to assess the sensitivity of three types of pancreatic cancer cells (Panc-1, BxPC-3 and T3-M4) to gemcitabine (Fig. 1A). The results showed that BxPC-3 was the most sensitive among the PDAC cells. Furthermore, we detected eIF5A2 protein and mRNA expression levels by western blot and RT-qPCR analyses, respectively. We observed the highest protein and mRNA expression levels of eIF5A2 in Panc-1 
A

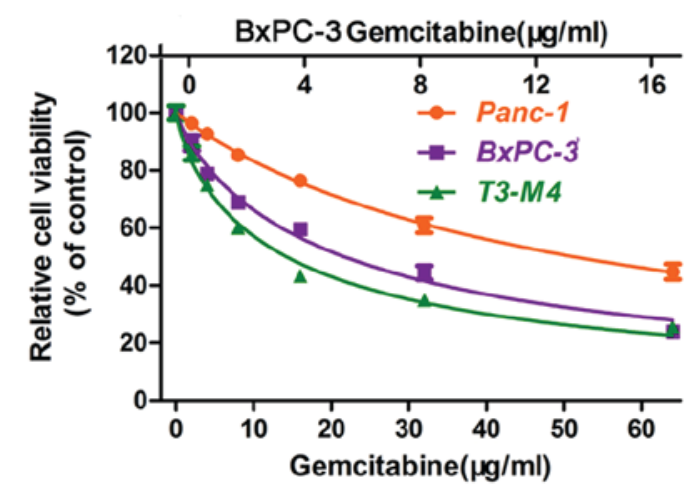

B

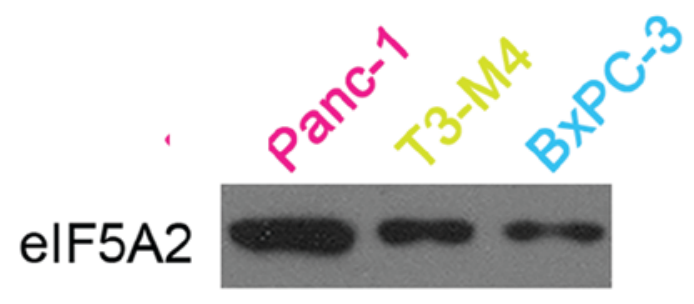

GAPDH
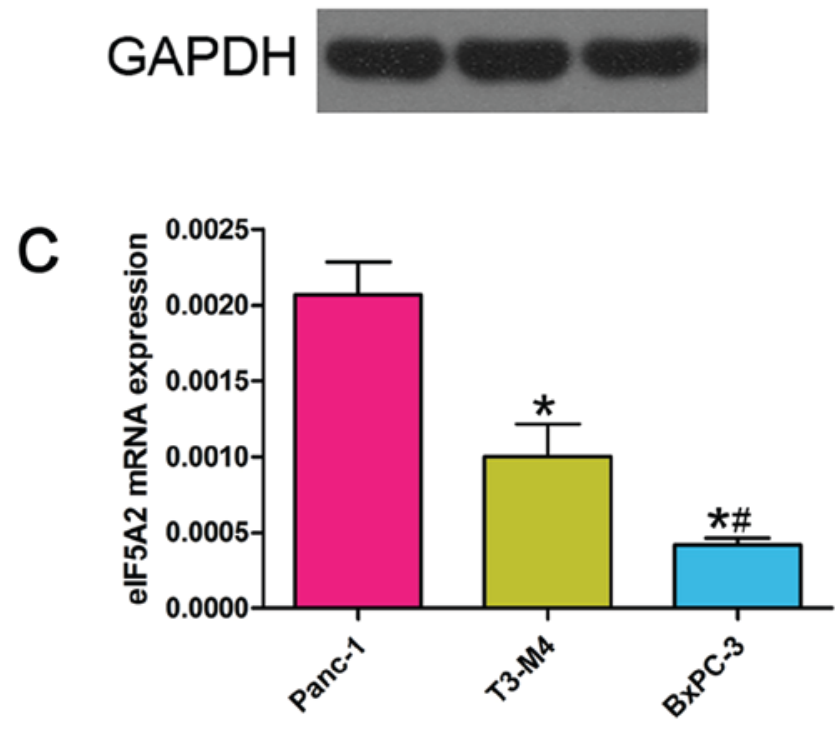

Figure 1. Different concentration of gemcitabine sensitivity in pancreatic cancer cell lines. (A) Cell Counting Kit-8 viability assay was used to examine sensitivity of different concentration of gemcitabine in pancreatic cancer cells. (B) Western blotting was used to detect the expression of eIF5a 2 in pancreatic cancer cells treated with gemcitabine, with GAPDH as a loading control. (C) Expression levels of eIF-5A2 mRNA in PDAC cells were determined by reverse transcription-quantitative polymerase chain reaction. ${ }^{*} \mathrm{P}<0.05$ vs. Panc-1; ${ }^{\#} \mathrm{P}<0.05$ vs. T3-M4.

and the lowest expression levels in BxPC-3 (Fig. 1B and C), indicating that eIF5A2 may be involved in chemoresistance to gemcitabine.

Effect of gemcitabine combined with GC7 on the sensitivity of PDAC cells. To investigate the synergistic effects of gemcitabine combined with GC7, we initially measured the effect of GC7 on PDAC cell viability. A series of GC7 concentrations ranging from 0-64 $(\mu \mathrm{M})$ were used for PDAC cell incubation. High concentrations of GC7 $(64 \mu \mathrm{M})$ significantly suppressed the viability of PDAC cells $(\mathrm{P}<0.05$;
Table I. $\mathrm{IC}_{50}$ values for gemcitabine in PDAC cell lines with or without GC7 $(16 \mu \mathrm{M})$ treatment.

\begin{tabular}{lrc}
\hline & \multicolumn{2}{c}{$\mathrm{IC}_{50}(\mu \mathrm{g} / \mathrm{ml})$} \\
\cline { 2 - 3 } PDAC cell line & Gemcitabine & Gemcitabine+GC7 \\
\hline Panc-1 & $45.95 \pm 1.54$ & $23.46 \pm 0.59^{\mathrm{a}}$ \\
BxPC-3 & $5.47 \pm 0.18$ & $2.74 \pm 0.14^{\mathrm{a}}$ \\
T3-M4 & $11.66 \pm 0.33$ & $6.60 \pm 0.40^{\mathrm{a}}$ \\
\hline
\end{tabular}

${ }^{\mathrm{a}} \mathrm{P}<0.05$ vs. gemcitabine alone. PDAC, pancreatic ductal adenocarcinoma; $\mathrm{IC}_{50}$, half maximal inhibitory concentration.

Fig. 2A-C). Therefore, we hypothesized that $32 \mu \mathrm{M}$ GC7 was a suitable concentration for further combination with gemcitabine. We used CCK-8 assays to examine cell viability treated with gemcitabine alone or co-administered with GC7. Results indicated that GC7 significantly enhanced the sensitivity of gemcitabine in PDAC cell lines $(\mathrm{P}<0.05$; Fig. 2D-F; Table I).

GC7 enhances gemcitabine sensitivity via theinhibition of eIF5A2 in PDAC cells. We hypothesized that the expression of eIF5A2 is associated with gemcitabine sensitivity. In order to confirm this, we transfected eIF5A2 siRNA into PDAC cells. siRNA knockdown efficiency was examined by western blot analysis. The results indicated that GC7 was involved in gemcitabine sensitivity. Relative protein expression was quantified by band density with GAPDH serving as an internal control (Fig. 3A). CCK-8 viability assay was used to measure the viability of PDAC cells (Fig. 3B-D). The results indicated that siRNA-mediated silencing of eIF5A2 enhanced gemcitabine sensitivity of PDAC cells compared with negative siRNA. These transfected cells were subsequently treated with gemcitabine alone or gemcitabine plus GC7, prior to conducting CCK-8 assays. The results indicated that there was no significant difference in gemcitabine sensitivity between the two groups (Fig. 3E-G).

GC7 reverses gemcitabine-induced MDR1 in PDAC cells. MDR1 is an important mediator of drug resistance in many types of cancer (21). To elucidate the mechanism by which GC7 enhanced gemcitabine sensitivity, we investigated whether MDR1 is involved in gemcitabine resistance in PDAC cells. We assessed MDR1 expression levels using western blot analysis. The results indicated that MDR 1 was upregulated by treatment with gemcitabine but gemcitabine-induced MDR1 upregulation was downregulated by GC7 (Fig. 4A); eIF5A2 exhibited the same trend (Fig. 4B). These data suggested that MDR1 mediated the resistance to gemcitabine in PDAC cells and that GC7 enhanced the therapeutic efficacy of gemcitabine by downregulating MDR1 expression.

\section{Discussion}

PDAC is one of the most lethal malignancies with a particularly poor patient prognosis (22) as chemotherapeutic treatment for 
A

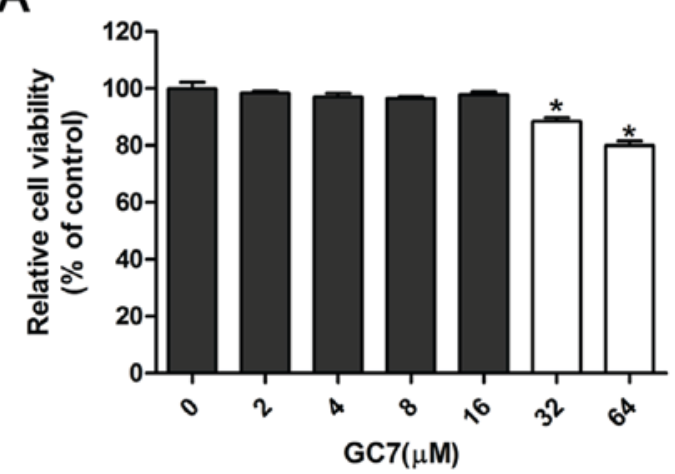

C

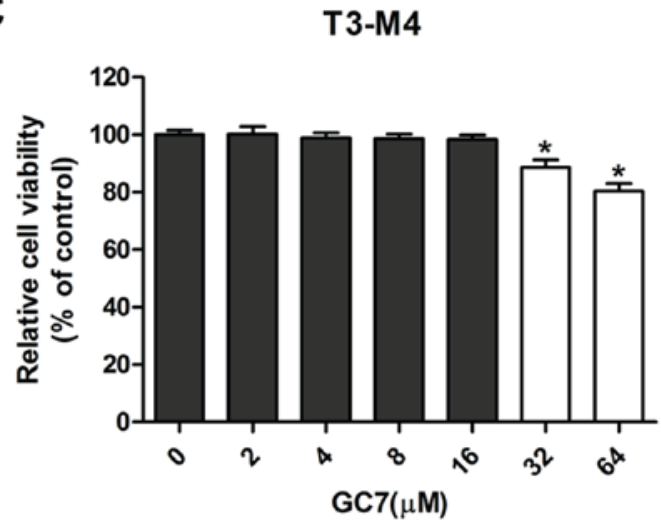

E

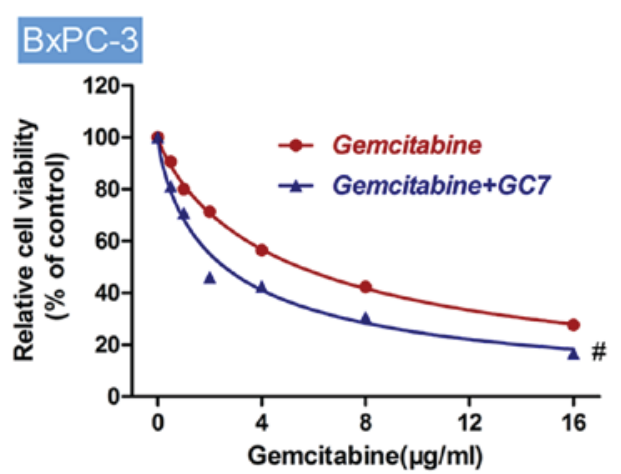

B

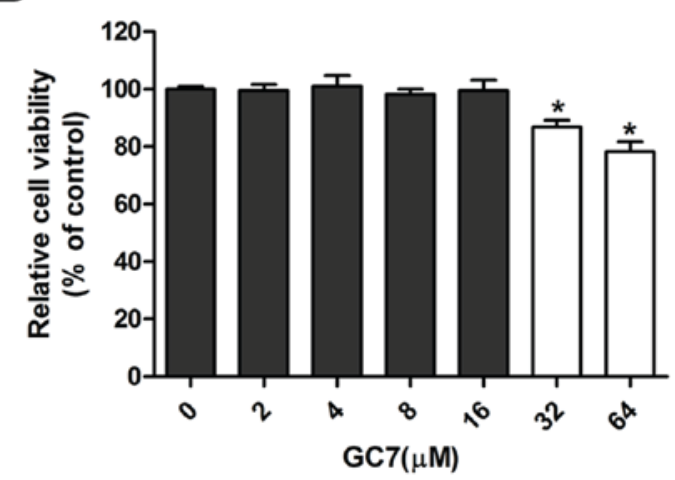

D Panc-1

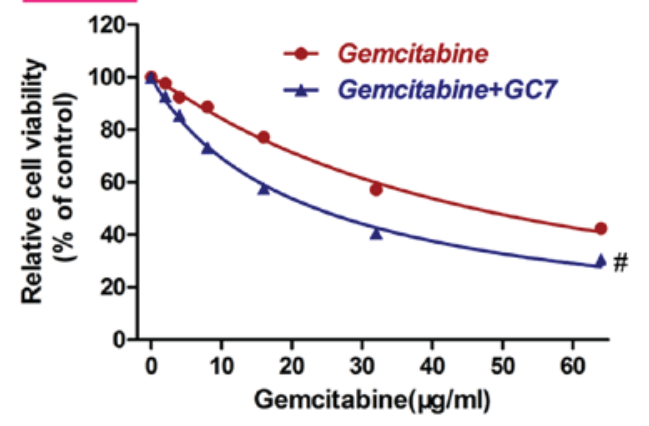

F

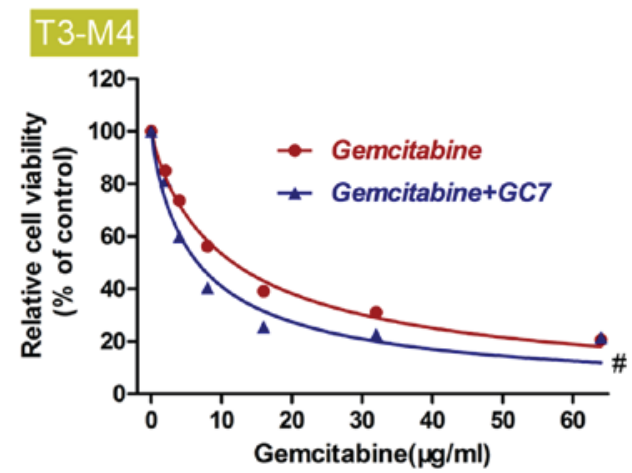

Figure 2. Effect of gemcitabine combined with GC7 on the sensitivity of pancreatic cancer cell lines. (A-C) Pancreatic cancer cell lines (Panc-1, BxPc3 and T3-M4) were incubated with different concentrations of GC7 over a period of $48 \mathrm{~h}$. Cell Counting Kit- 8 values of the treated pancreatic cancer cells were normalized to the control group with the absence of GC7. ${ }^{*} \mathrm{P}<0.05$ vs. $0 \mu \mathrm{M}$ GC7. (B) Western blot analysis was performed to determine eIF5A2 expression in pancreatic cancer cells treated with GC7. Relative protein expression was quantified by band density with GAPDH serving as an internal control. (D-F) Cell viability of pancreatic cancer cells after treatment with gemcitabine or gemcitabine combined with GC7. Cell viability was determined using the Cell Counting Kit-8 assay method. " $\mathrm{P}<0.05$ vs. Gemcitabine.

this type of cancer typically leads to drug resistance, which is a major obstacle in chemotherapy success. As a standard chemotherapeutic drug for the treatment of PDAC cells, gemcitabine alone or in combination with other compounds rarely improves survival in patients in the advanced stages of this cancer (23). Therefore, the identification of novel markers to predict gemcitabine resistance related to chemoresistance is high research priority for PDAC treatment. In this study, we found that gemcitabine combined with GC7 significantly increased the therapeutic efficacy of gemcitabine in PDAC, suggesting the potential clinical application of GC7 in patients who have acquired resistance to gemcitabine.
eIF5A2, which is an essential component of translation elongation, has an important role in cell proliferation, metastasis, and apoptosis, and it has recently been considered as a novel oncogene (13). GC7, an inhibitor of eIF5A2 activation, exhibits obvious anti-tumor effects in human cancer, including hepatocellular carcinoma and glioblastoma $(12,24)$. In this study, we measured the expression levels of eIF5A2 in three PDAC cell lines and showed cell line variation at the protein levels. Furthermore, we found that higher expression levels correlated with a decreased sensitivity to gemcitabine, whereas exposure to gemcitabine increased eIF5A2 expression. Consequently, we postulated that overexpression of 
A

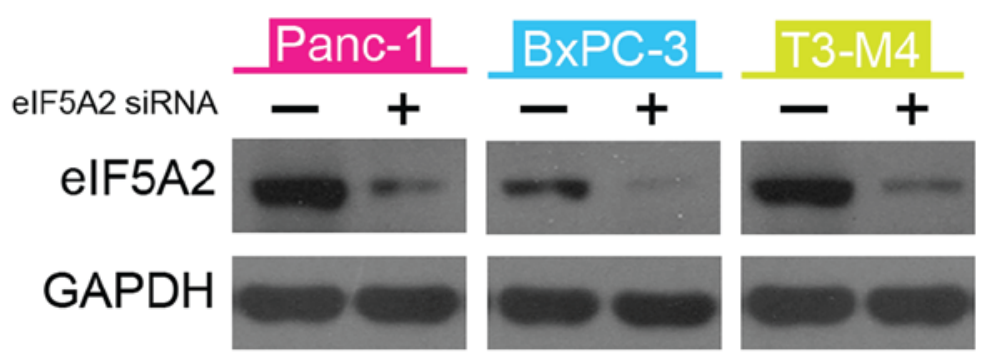

B Panc-1

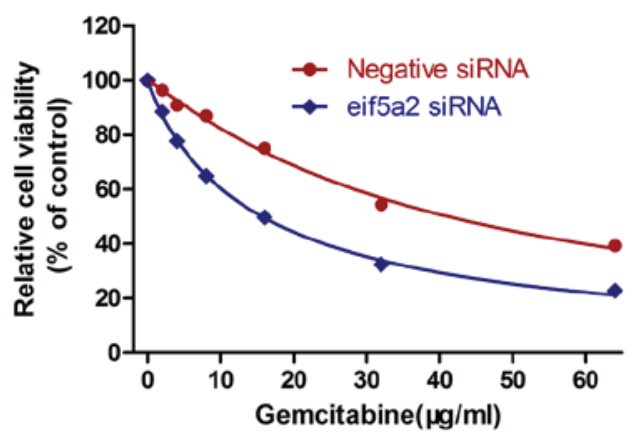

D

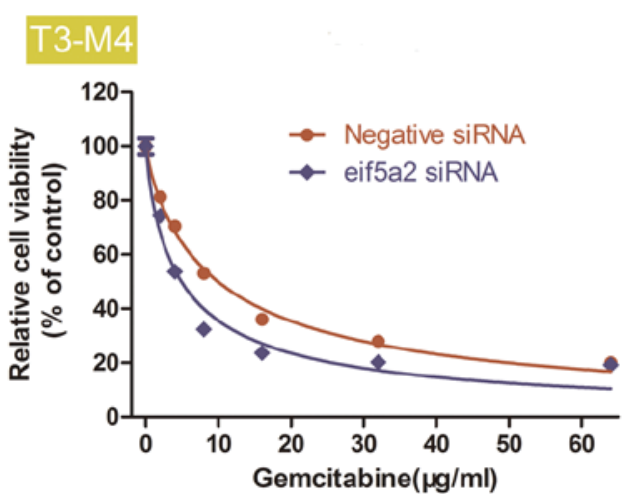

$\mathrm{F}$

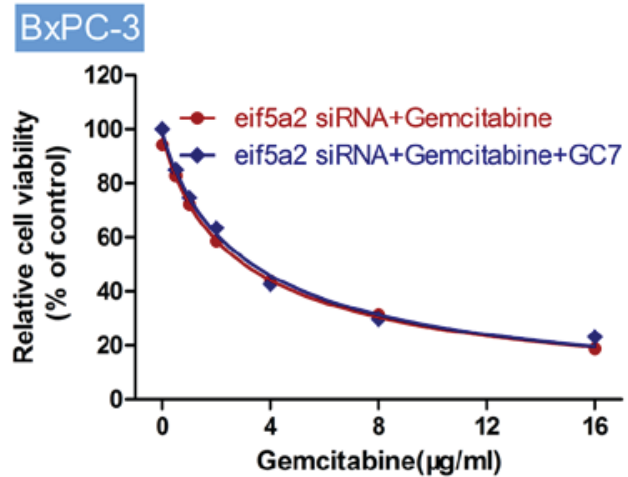

C

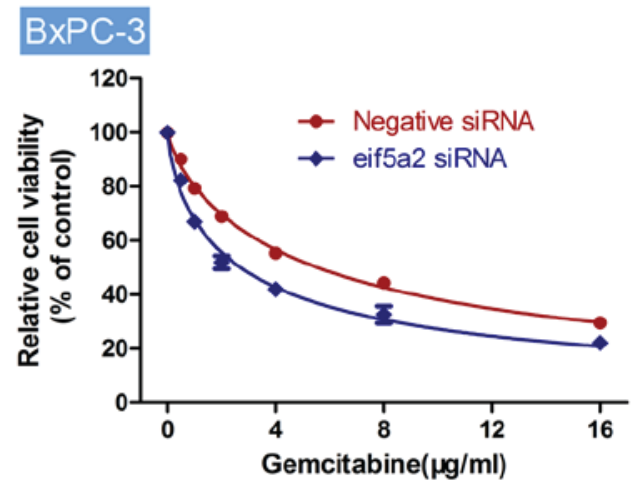

E Panc-1

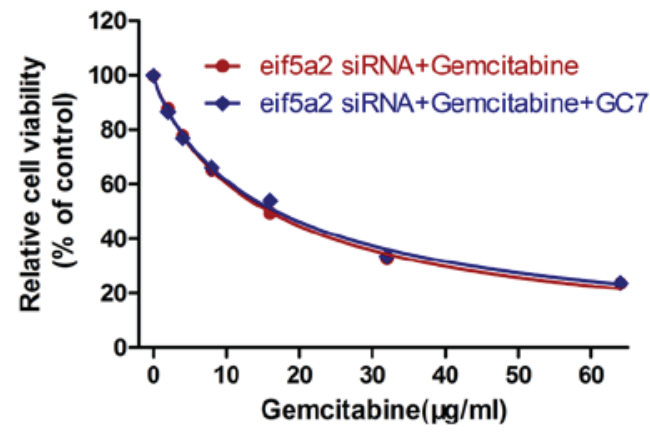

G

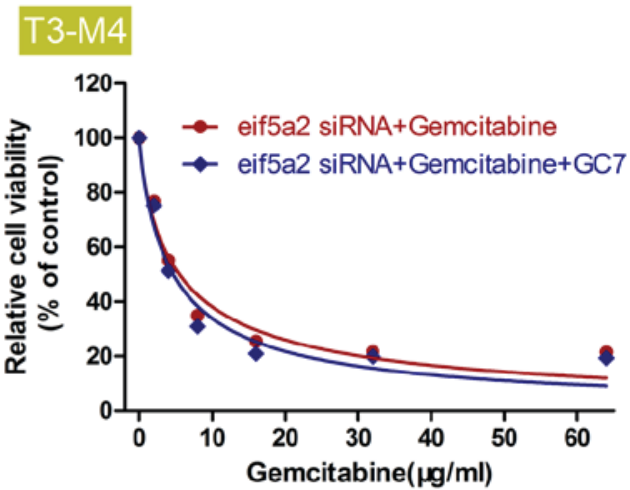

Figure 3. GC7 enhances gemcitabine sensitivity through inhibition of eIF5A2 in PDAC cells. (A) Western blot analysis for the expression of eIF5A2 in eIF5A2 siRNA-transfected cells. GAPDH was used as an internal control. (B-D) Cell Counting Kit-8 viability assay showing knockdown of eIF5A2 increasing gemcitabine sensitivity of pancreatic cancer cells. (E-G) Following knockdown of eIF5A2 using siRNA, Cell Counting Kit-8 cell viability assays were used to determine the sensitivity of gemcitabine or gemcitabine plus GC7 in pancreatic cancer cells treated with eIF5A2 siRNA.

eIF5A2 was associated with drug resistance and that overexpression of eIF5A2 was a trait of various cancers that are likely to become drug resistant. To test this hypothesis, we investigated whether GC7 suppressed eIF5A2, thereby promoting 

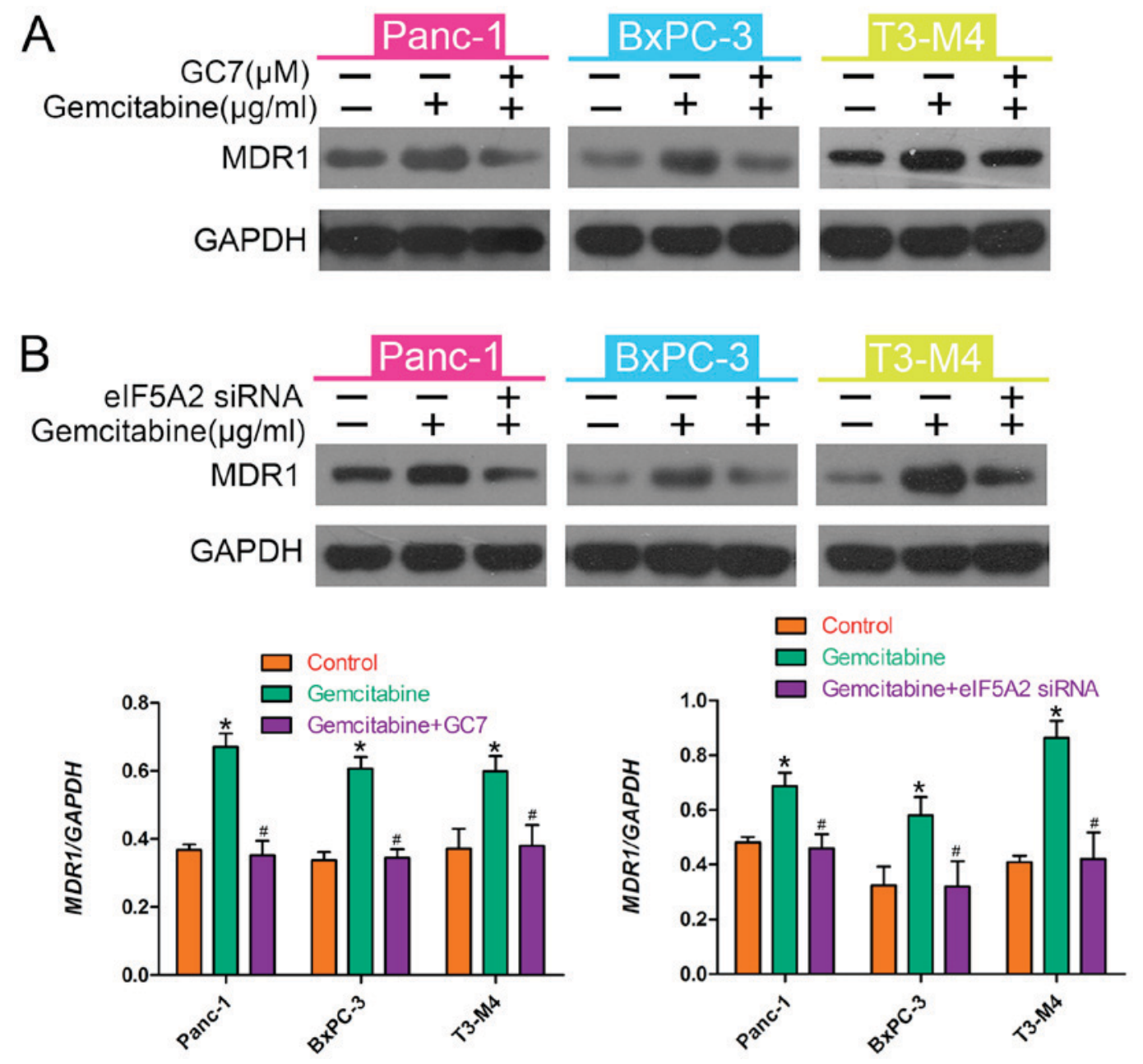

Figure 4. GC7 reverses gemcitabine-induced MDR1 in PDAC cells. (A) Western blot analysis was used to detect eIF5A2 expression in pancreatic cancer cells treated with gemcitabine, or gemcitabine in combination with GC7. Relative protein expression levels in PDAC cells were quantified by band density with GAPDH serving as a loading control. (B) Western blotting was used to detect eIF5A2 expression in pancreatic cancer cells treated with gemcitabine or gemcitabine in combination with siRNAs for eIF5A2. ${ }^{\mathrm{P}}<0.05$ vs. control; ${ }^{*} \mathrm{P}<0.05$ vs. gemcitabine.

the therapeutic efficacy of gemcitabine against PDAC cells. The results indicated that treatment of GC7 plus gemcitabine in PDAC cells significantly inhibited cell viability. eIF5A2 siRNA was also transfected into PDAC cells to knockdown the expression of eIF5A2. The results showed that there was no significant difference in gemcitabine sensitivity between cells treated with gemcitabine alone or with gemcitabine plus GC7. A recent study demonstrated that combined treatment with GC7 enhanced the therapeutic efficacy of doxorubicin by inhibiting epithelial-mesenchymal transition in hepatocellular carcinoma cells and bladder cancer cells $(25,26)$ whereas enhancing cetuximab sensitivity by inhibiting eIF5A2 has been demonstrated in non-small cell lung cancer (NSCLC) cells (27). In addition, cisplatin sensitivity was enhanced in NSCLC cells after incubation with GC7 (28). Therefore, data from the current study showed that co-treatment with gemcitabine and GC7 may be a potent therapeutic strategy against PDAC cells.

Furthermore, we investigated the molecular mechanism underlying GC7 enhancement of sensitivity to gemcitabine in PDAC cells. Previous studies have revealed that overexpression of the MDR gene may be responsible for resistance to chemotherapy $(29,30)$. Our study showed that GC7 downregulated MDR1 expression where gemcitabine had induced the expression of MDR1. Furthermore, a decrease in MDR1 enhanced the inhibitory effect of gemcitabine on cell viability. These observations suggest that MDR1 is an important mediator of drug resistance in various types of cancer such as breast cancer, colon cancer and leukemia cells (21). Therefore, we postulated that MDR1 mediated gemcitabine-induced drug resistance and influenced its therapeutic effect in PDAC cells. MDR1 maybe a target for PDAC therapy through a combination of gemcitabine with GC7. In this study, GC7 enhanced the sensitivity of PDAC cells to gemcitabine by suppressing MDR1 expression, suggesting that MDR1 may be a target for PDAC therapy.

In conclusion, the findings of the present study demonstrated that combined gemcitabine treatment with GC7 significantly enhanced the cytotoxicity of gemcitabine in PDAC cells via the inhibition of eIF5A2, thereby preventing gemcitabine-induced MDR1. Therefore, it is possible that combination therapy with GC7 may contribute to improved clinical outcomes in gemcitabine-based chemotherapy for PDAC patients. 


\section{References}

1. Malvezzi M, Bertuccio P, Levi F, La Vecchia C and Negri E: European cancer mortality predictions for the year 2014. Ann Oncol 25: 1650-1656, 2014.

2. American Cancer Society: Cancer Facts \& Figures 2015. Atlanta: American Cancer Society; 2015.

3. Gong Z, Holly EA and Bracci PM: Survival in population-based pancreatic cancer patients: San Francisco Bay area, 1995-1999. Am J Epidemiol 174: 1373-1381, 2011.

4. Siegel RL, Miller KD and Jemal A: Cancer statistics, 2015. CA Cancer J Clin 65: 5-29, 2015.

5. Rahib L, Smith BD, Aizenberg R, Rosenzweig AB, Fleshman JM and Matrisian LM: Projecting cancer incidence and deaths to 2030: The unexpected burden of thyroid, liver, and pancreas cancers in the United States. Cancer Res 74: 2913-2921, 2014.

6. Paulson AS, Tran Cao HS, Tempero MA and Lowy AM: Therapeutic advances in pancreatic cancer. Gastroenterology 144: 1316-1326, 2013.

7. Zhu W, Cai MY, Tong ZT, Dong SS, Mai SJ, Liao YJ, Bian XW, Lin MC, Kung HF, Zeng YX, et al: Overexpression of EIF5A2 promotes colorectal carcinoma cell aggressiveness by upregulating MTA1 through C-myc to induce epithelial-mesenchymaltransition. Gut 61: 562-575, 2012.

8. Cao D, Hustinx SR, Sui G, Bala P, Sato N, Martin S, Maitra A, Murphy KM, Cameron JL, Yeo CJ, et al: Identification of novel highly expressed genes in pancreatic ductal adenocarcinomas through a bioinformatics analysis of expressed sequence tags. Cancer Biol Ther 3: 1081-1091, 2004.

9. Fujimura K, Wright T, Strnadel J,Kaushal S, Metildi C, Lowy AM, Bouvet M, Kelber JA and Klemke RL: A hypusine-eIF5A-PEAK1 switch regulates the pathogenesis of pancreatic cancer. Cancer Res 74: 6671-6681, 2014.

10. Yang GF, Xie D, Liu JH, Luo JH, Li LJ, Hua WF, Wu HM, Kung HF, Zeng YX and Guan XY: Expression and amplification of eIF-5A2 in human epithelial ovarian tumors and overexpression of EIF-5A2 is a new independent predictor of outcome in patients with ovarian carcinoma. Gynecol Oncol 112: 314-318, 2009.

11. Wang FW, Cai MY, Mai SJ, Chen JW, Bai HY, Li Y, Liao YJ, Li CP, Tian XP, Kung HF, et al: Ablation of EIF5A2 induces tumor vasculature remodeling and improves tumor response to chemotherapy via regulation of matrix metalloproteinase 2 expression. Oncotarget 5: 6716-6733, 2014.

12. Tang DJ, Dong SS, Ma NF, Xie D, Chen L, Fu L, Lau SH, Li Y, Li Y and Guan XY: Overexpression of eukaryotic initiation factor 5A 2 enhances cell motility and promotes tumor metastasis in hepatocellular carcinoma. Hepatology 51: 1255-1263, 2010.

13. He LR, Zhao HY, Li BK, Liu YH, Liu MZ, Guan XY, Bian XW, Zeng YX and Xie D: Overexpression of eIF5A-2 is an adverse prognosticmarker of survival in stage I non-small cell lung cancer patients. Int J Cancer 129: 143-150, 2011.

14. Li Y, Fu L, Li JB, Qin Y, Zeng TT, Zhou J, Zeng ZL, Chen J, Cao TT, Ban X, et al: Increased expression of EIF5A2, via hypoxia or gene amplification, contributes to metastasis and angiogenesis of esophageal squamous cell carcinoma. Gastroenterology 146: 1701-1713.e9, 2014.

15. Meng QB, Kang WM, Yu JC, Liu YQ, Ma ZQ, Zhou L, Cui QC and Zhou WX: Overexpression of eukaryotic translation initiation factor 5A2 (EIF5A2) correlates with cell aggressiveness and poor survival in gastric cancer. PLoS One 10: e0119229, 2015.

16. Bao Y, Lu Y, Wang X, Feng W, Sun X, Guo H, Tang C, Zhang X Shi Q and $\mathrm{Yu} \mathrm{H}$ : Eukaryotic translation initiation factor 5A2 (eIF5A2) regulates chemoresistance in colorectal cancer through epithelial mesenchymal transition. Cancer Cell Int 15: 109, 2015.
17. Khosravi S, Wong RP, Ardekani GS, Zhang G, Martinka M, Ong CJ and Li G: Role of EIF5A2, a downstream target of Akt, in promoting melanoma cell invasion. Br J Cancer 110: 399-408, 2013.

18. Wang FW, Guan XY and Xie D: Roles of eukaryotic initiation factor 5A2 in human cancer. Int J Biol Sci 9: 1013-1020, 2013.

19. Liu Y, Liu R, Fu P, Du F, Hong Y, Yao M, Zhang X and Zheng S: N1-Guanyl-1,7-Diaminoheptane sensitizes estrogen receptor negative breast cancer cells to doxorubicin by preventing epithelial-mesenchymal transition through inhibition of eukaryotic translation initiation factor 5A2 activation. Cell Physiol Biochem 36: 2494-2503, 2015.

20. Tuo YL, Li XM and Luo J: Long noncoding RNA UCA1 modulates breast cancer cell growth and apoptosis through decreasing tumor suppressive miR-143. Eur Rev Med Pharmacol Sci 19: 3403-3411, 2015

21. Kartal-Yandim M, Adan-Gokbulut A and Baran Y: Molecular mechanisms of drug resistance and its reversal in cancer. Crit Rev Biotechnol 36: 716-726, 2016.

22. Ryan DP, Hong TS and Bardeesy N: Pancreatic adenocarcinoma. N Engl J Med 371: 1039-1049, 2014.

23. Stathis A and Moore MJ: Advanced pancreatic carcinoma: Current treatment and future challenges. Nat Rev Clin Oncol 7: 163-172, 2010.

24. Preukschas M, Hagel C, Schulte A, Weber K, Lamszus K, Sievert H, Pällmann N, Bokemeyer C, Hauber J, Braig M and Balabanov S: Expression of eukaryotic initiation factor $5 \mathrm{~A}$ and hypusine forming enzymes in glioblastoma patient samples: Implications for new targeted therapies. PLoS One 7: e43468, 2012.

25. Lou B, Fan J, Wang K, Chen W, Zhou X, Zhang J, Lin S, Lv F and Chen Y: N1-guanyl-1,7-diaminoheptane (GC7) enhances the therapeutic efficacy of doxorubicin by inhibiting activation of eukaryotic translation initiation factor 5A2 (eIF5A2) and preventing the epithelial-mesenchymal transition in hepatocellular carcinoma cells. Exp Cell Res 319: 2708-2717, 2013.

26. Yang J, Yu H, Shen M, Wei W, Xia L and Zhao P: N1guanyl-1,7-diaminoheptane sensitizes bladder cancer cells to doxorubicin by preventing epithelial-mesenchymal transition through inhibition of eukaryotic translation initiation factor 5A2 activation. Cancer Sci 105: 219-227, 2014.

27. Wang X, Jiang R, Cui EH, Feng WM, Guo HH, Gu DH, Tang CW, Xue T and Bao Y: N1-guanyl-1,7-diaminoheptane enhances the chemosensitivity of NSCLC cells to cetuximab through inhibition of eukaryotic translation initiation factor $5 \mathrm{~A} 2$ activation. Eur Rev Med Pharmacol Sci 20: 1244-1250, 2016.

28. Xu G, Yu H, Shi X, Sun L, Zhou Q, Zheng D, Shi H, Li N, Zhang X and Shao G: Cisplatin sensitivity is enhanced in non-small cell lung cancer cells by regulating epithelial-mesenchymal transition through inhibition of eukaryotic translation initiation factor 5A2. BMC Pulm Med 14: 174, 2014.

29. Li QQ, Xu JD, Wang WJ, Cao XX, Chen Q, Tang F, Chen ZQ, Liu XP and Xu ZD: Twist1-mediated adriamycin-induced epithelial-mesenchymal transition relates to multidrug resistance and invasive potential in breast cancer cells. Clin Cancer Res 15: 2657-2665, 2009.

30. Saxena M, Stephens MA, Pathak H and Rangarajan A: Transcription factors that mediate epithelial-mesenchymal transition lead to multidrug resistance by upregulating $\mathrm{ABC}$ transporters. Cell Death Dis 2: e179, 2011. 